\title{
“Carga de diazepam”: ¿puede una estrategia para prevenir abstinencia al alcohol usarse con éxito para tratar la dependencia a benzodiacepinas?
}

\section{"Diazepam loading": ¿Can a strategy for preventing alcohol withdrawal be used to treat benzodiazepine use disorder?}

\author{
Clara Oliveras*, Adriana Fortea*, Laura Espinosa*, Pablo Barrio*, \\ Anna Lligoña*, Mercè Balcells-Olivero*. \\ * Addictions Unit. Psychiatry Department. Clinical Neuroscience Institute. \\ Hospital Clínic i Universitari de Barcelona. Barcelona. Spain.
}

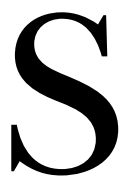

r. Editor,

Las benzodiacepinas (BZDs) son fármacos depresores del sistema nervioso central (SNC) ampliamente utilizados en la población para el tratamiento del insomnio y la ansiedad, a pesar de tener efectos secundarios adversos a largo plazo (Fortea González, Oriolo, Balcells Oliveró, Sánchez Del Valle y Castellvi, 2017). De forma similar al alcohol, están implicadas en fenómenos de tolerancia y dependencia tras un consumo continuado. La discontinuación en estos casos puede producir síntomas abstinenciales como temblor, ansiedad, convulsiones y, ocasionalmente, la muerte (Brett y Murnion, 2015).

El diazepam es una BZD empleada para tratar y prevenir la abstinencia alcohólica (Bird y Makela, 1994), así como la abstinencia a BZDs. El diazepam tiene una vida media de eliminación larga (20-100 horas) al igual que su metabolito hepático activo, el nordiazepam (36-200 horas) (Greenblatt, Shader, Divoll y Harmatz, 1981), lo que hace posible que existan de forma prolongada concentraciones terapéuticas incluso después de tratamientos de corta duración (Muzyk, Leung, Nelson, Embury y Jones, 2013). Tiene además una naturaleza altamente lipofílica que le permite un rápido inicio de acción, alcanzando rápidamente su concentración máxima en el SNC.

Entre las estrategias para el abordaje de la abstinencia alcohólica, se considera válido el empleo de dosis de carga de BZDs (Lligoña, 2007; Wasilewski et al., 1996). El perfil farmacocinético del diazepam permite cargar inicialmente una dosis elevada para conseguir un efecto de tratamiento inmediato y sostenido. Normalmente esta carga consiste en administrar $20 \mathrm{mg}$ de diazepam por vía oral cada 2 horas hasta que el paciente esté somnoliento pero reactivo. Posteriormente, existirán concentraciones terapéuticas de diazepam y nordiazepam hasta pasadas las 72 horas.

Sin embargo, pese a sus propiedades farmacológicas y su uso para abstinencia alcohólica, la estrategia de carga no ha sido reportada en la literatura para el tratamiento de la abstinencia a BZDs, salvo como estrategia inicial en caso de sintomatología abstinencial aguda seguida de una pauta descendente lenta (Sellers, 1988).

Describimos el caso de una mujer de 51 años con antecedentes de trastorno por uso de BZDs de larga evolución que fue desintoxicada con éxito después de recibir una dosis de carga de diazepam sin posterior administración de benzodiazepinas.

Entre su historia toxicológica destaca abuso de cocaína esnifada y cannabis en el pasado. Mantenía consumo habitual de tabaco y esporádico de alcohol. Entre sus antecedentes destaca trastorno de personalidad, con predominio de rasgos histriónicos y límites (DSM-5), y conductas autolesivas.

El consumo de BZDs fue iniciado por prescripción médica en relación a ataques de pánico, hasta posteriormente desarrollar un patrón de dependencia. Realizó dos ingresos previos para desintoxicación, empleándose pauta descendente de clonazepam, con posterior recaída. En la ac-

Recibido: Octubre 2017; Aceptado: Noviembre 2017.

Enviar correspondencia a:

Clara Oliveras. Addictions Unit. Psychiatry Department. Clinical Neuroscience Institute. Hospital Clínic i Universitari de Barcelona. Calle Villarroel 170. 08036 Barcelona. Spain. Teléfono: +34669646380. E-mail: coliveras@clinic.cat. 


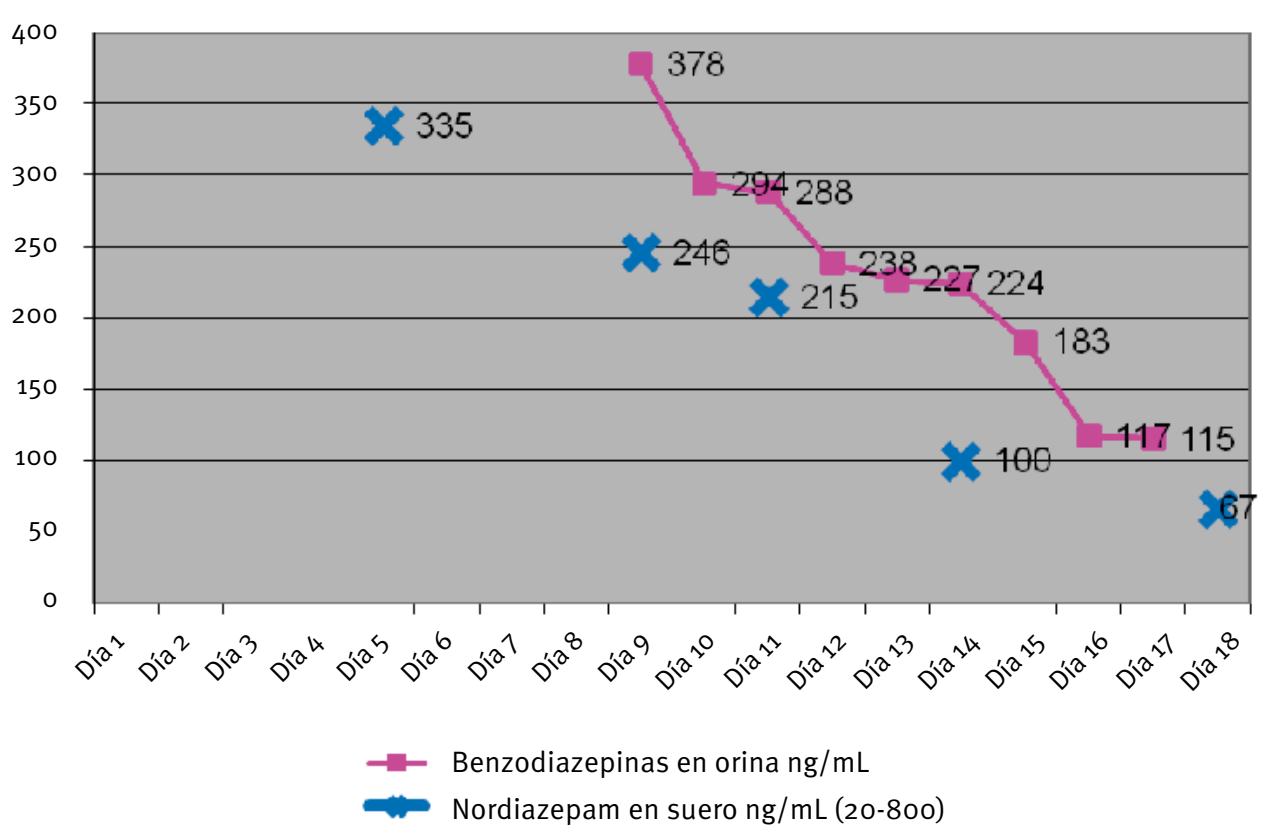

Figura 1. Monitorización de los niveles de benzodiazepinas en orina y nordiazepam en sangre (entre paréntesis se indica el intervalo considerado terapéutico por el laboratorio) en el tiempo de ingreso. Se aprecia presencia de niveles terapéuticos de metabolito activo en sangre durante un período prolongado.

tualidad presentaba consumo diario de $5 \mathrm{mg}$ de lorazepam y $100 \mathrm{mg}$ de diazepam.

La paciente es trasladada a urgencias tras sobreingesta estimada de $200 \mathrm{mg}$ de diazepam con finalidad suicida. La prueba de tóxicos en orina fue negativa para opioides y etanol. Durante el período de observación presentó depresión respiratoria, por lo que se le administró perfusión intravenosa de flumazenilo durante 8 horas. No se administró carbón activado. Posteriormente fue ingresada en la Unidad de Agudos de Psiquiatría.

Dado que el flumazenilo suprime los efectos de las BZDs por inhibición pero no las elimina del organismo, se decidió usar una estrategia de carga de diazepam análoga a la usada para la desintoxicación alcohólica para tratar el síndrome de abstinencia a BZDs de la paciente. Se consideró dosis de carga la ingesta previa de $200 \mathrm{mg}$ de diazepam.

No experimentó síntomas ni signos graves de abstinencia a BZDs tales como delirium o convulsiones. Más allá de ansiedad leve que remitió gradualmente, tampoco se objetivó ni refirió espontáneamente ningún otro signo ni síntoma de abstinencia ni precisó dosis de rescate. Al cuarto día se inició monitorización de BZDs en sangre y orina. Fue dada de alta a los 18 días. Tras 24 semanas, mantenía abstinencia.

En nuestra experiencia, y de forma congruente con su uso para la abstinencia alcohólica, la carga única de diazepam puede ser una estrategia válida para la prevención y tratamiento de la abstinencia a BZD. La vida media del diazepam y sus metabolitos (como nordiazepam) permiten mantener niveles séricos de sustancia activa elevados, facilitando su reducción progresiva en el tiempo (Figura 1) y evitando así la aparición de clínica abstinencial. De esta manera se evita la administración de nuevas dosis de BZDs y no se refuerza la conducta de búsqueda de sustancia como sucedería cuando las BZDs se administran en dosis fraccionadas durante varios días.

Si bien en nuestro caso se consideró la propia sobreingesta realizada por la paciente como dosis de carga, ésta se podría efectuar de forma supervisada para evitar complicaciones derivadas de la misma. Así, aunque se precisen nuevos estudios para evaluar la seguridad, dosis de carga y eficacia respecto a estrategias convencionales de tratamiento y prevención de la abstinencia a BZDs, la dosis de carga de diazepam podría ser una alternativa eficaz que además podría minimizar la recaída en el consumo.

\section{Conflicto de interés}

Los autores declaran que no tienen ningún conflicto de interés.

\section{Referencias}

Bird, R. D. y Makela, E. H. (1994). Alcohol withdrawal: what is the benzodiazepine of choice? The Annals of Pharmacotherapy, 28, 67-71. doi:10.1177/106002809402800114. 
Brett, J. y Murnion, B. (2015). Management of benzodiazepine misuse and dependence. Australian Prescriber, 38, 152-155. doi:10.18773/austprescr.2015.055.

Fortea González, A., Oriolo, G., Balcells Oliveró, M., Sanchez del Valle, R. y Castellvi, M. (2017). Deterioro cognitivo secundario a trastorno por uso de benzodiacepinas y su reversibilidad: a propósito de un caso. Adicciones, 29, 61-63. doi:10.20882/adicciones.767.

Greenblatt, D. J., Shader, R. I., Divoll, M. y Harmatz, J. S. (1981). Benzodiazepines: a summary of pharmacokinetic properties. British Journal of Clinical Pharmacology, 11, 11S-16S. doi:10.1111/j.1365-2125.1981.tb01833.x.

Lligoña, A. (2007). Tratamiento de la abstinencia alcohólica con benzodiacepinas. Una revisión. Aula Médica Psiquiatría, 2, 65-77.

Muzyk, A. J., Leung, J. G., Nelson, S., Embury, E. R. y Jones, S. R. (2013). The role of diazepam loading for the treatment of alcohol withdrawal syndrome in hospitalized patients. The American Journal on Addictions, 22, 113-118. doi:10.1111/j.1521-0391.2013.00307.x.

Sellers, E. M. (1988). Alcohol, barbiturate and benzodiazepine withdrawal syndromes: Clinical management. $\mathrm{Ca}$ nadian Medical Association Journal, 139, 113-120.

Wasilewski, D., Matsumoto, H., Kur, E., Dziklińska, A., Woźny, E., Stencka, K., ... Szelenberger, W. (1996). Assessment of diazepam loading dose therapy of delirium tremens. Alcohol and Alcoholism, 31, 273-278. 